\title{
Linx
}

Revue des linguistes de l'université Paris X Nanterre

40 | 1999

Le statut d'unité lexicale

\section{La notion d' " unité lexicale » en linguistique et son usage en lexicologie}

The notion of "lexical unit" in linguistics and its use in lexicology.

\section{Fabienne Cusin-Berche}

\section{CpenEdition}

1 Journals

Édition électronique

URL : http://journals.openedition.org/linx/730

DOI : $10.4000 / \operatorname{linx} .730$

ISSN : 2118-9692

Éditeur

Presses universitaires de Paris Nanterre

\section{Édition imprimée}

Date de publication : 1 juin 1999

Pagination : 11-10

ISSN : 0246-8743

\section{Référence électronique}

Fabienne Cusin-Berche, "La notion d' " unité lexicale » en linguistique et son usage en lexicologie », Linx [En ligne], 40 | 1999, mis en ligne le 22 juin 2012, consulté le 10 décembre 2020. URL : http:// journals.openedition.org/linx/730 ; DOI : https://doi.org/10.4000/linx.730

Ce document a été généré automatiquement le 10 décembre 2020.

Département de Sciences du langage, Université Paris Ouest 


\title{
La notion d' « unité lexicale » en linguistique et son usage en lexicologie
}

The notion of «lexical unit » in linguistics and its use in lexicology.

\author{
Fabienne Cusin-Berche
}

1 L'ambiguïté du syntagme nominal unité lexicale incite à faire le point sur la notion afférente à cette dénomination comparativement à celles qui lui sont concurrentes dans les sciences du langage - telles que mot, terme, lexème, vocable - avant d'examiner, dans une perspective strictement lexicologique, les procédures d'accès à ce statut et d'explorer, de ce fait, les manifestations et les facteurs de lexicalisation.

2 Une investigation de ce type portant sur l'objet d'études du lexicologue convie, en outre, à un questionnement plus général sur la place de sa discipline au sein de la linguistique et par rapport à des domaines connexes comme la lexicographie et la terminologie. Ainsi consultant un ouvrage de linguistique, récent, qui se veut généraliste, on s'aperçoit que la lexicologie semble avoir disparu du paysage scientifique :

« Nous proposons un découpage de la linguistique maintenant bien accepté. Nous distinguons les disciplines qui s'occupent de la forme de la langue (la phonologie, la morphologie et la syntaxe) de celles qui s'intéressent au sens (la sémantique et la pragmatique) » [Moeschler J. \& Auchlin A., $1997:$ 7]

3 Cette exclusion, qui pourrait se justifier par le fait que la discipline en question ne se plie pas aisément à la partition «forme vs sens ", ne peut être corrélée avec l'élimination de toute référence à l'«unité lexicale», car celle-ci est évoquée pour expliciter les préoccupations de la démarche morphologique :

«La morphologie a pour objet la structure formelle des unités signifiantes de la langue (unités lexicales et grammaticales que sont les mots) » [idem]

4 Le terme générique adopté pour désigner ce qui ce qui se trouve entre le morphème (éventuellement inclus) et le syntagme est mot. Toutefois, l'énoncé parenthétique est ambigu puisqu'il est possible d'en déduire que les mots sont soit des unités lexicales soit des unités grammaticales - ce qui réactive une distinction traditionnelle - ou a contrario 
que les mots sont à la fois des unités lexicales et des unités grammaticales, ce qui revient à dire que tout mot relève du lexique et de la grammaire (ne serait-ce que par son appartenance grammaticale). Donc dans cette Introduction à la linguistique contemporaine [1997], le lexique ou la lexicologie n'apparaissent pas en tant que tels. Ce n'est qu'à partir de la morphologie (chapitre 5) que sont abordées les questions relatives aux différents types d'unités : il est alors question de mot, de morphème lexical, de mot lexical, de lexème, mais non de l'unité lexicale proprement dite. En revanche, le lexicologue s'intéresse à la morphologie ${ }^{1}$, à la sémantique, voire à la pragmatique, et sollicite les apports de la syntaxe, mais ses recherches ont une finalité spécifique : rendre compte de la constitution et du fonctionnement du système lexical tant d'un point de vue morphologique que sémiotique et sémantique.

5 L'absence de prise en compte de la lexicologie dans certains ouvrages pourrait suggérer que la discipline est en déclin et confirmer que «le lexique est le parent pauvre de la linguistique moderne » [Rey-Debove, 1998 : 193]. Toutefois, à la vue de la recrudescence de travaux relevant de ce champ - après la réédition du Précis de lexicologie de J. Picoche ${ }^{2}$, et la parution ces deux dernières années de trois manuels dressant un état des lieux sur la question ${ }^{3}$ - on est amené à partager le point de vue de D. Coste :

«Suivant les moments, les travaux relatifs au lexique paraissent ou non faire partie $\mathrm{du}$ domaine de la linguistique reconnue comme discipline scientifique, étant entendu que l'intérêt pour le lexique revêt lui-même, au fil de la période prise en compte, des formes différentes » [Coste D., 1988: 67].

\section{Les statuts de l'unité lexicale dans les travaux relatifs au lexique}

6 En dépit, donc, d'une certaine marginalisation scientifique, a surgi, paradoxalement, un regain d'intérêt à l'égard des études concernant le lexique, stimulées notamment par le dessein soit d'élaborer des dictionnaires électroniques plus performants ${ }^{4}$ que les ouvrages lexicographiques traditionnels, soit d'améliorer la transmission d'informations appartenant à des domaines d'activités scientifiques ou techniques. Ces travaux à visée dictionnairique et/ou terminologique, qui sont susceptibles de relever de la linguistique appliquée, sont très utiles au lexicologue, mais ils ne recouvrent pas totalement son propre champ d'investigation ${ }^{5}$.

7 Ainsi, dans le premier cas, est-ce à travers des problèmes de constitution de nomenclatures, i.e. d'organisation macro et microstructurelle, de liens hypertextuels, que pourrait réapparaitre la préoccupation d'une définition de ce qu'on entend par unité lexicale, mais la question est rarement abordée en ces termes, comme en témoignent $\mathrm{D}$. Le Pesant et M. Mathieu-Colas :

«Les unités lexicales ne peuvent être appréhendées comme des entités isolées, closes sur elles-mêmes, elles doivent au contraire être définies en termes d'emplois dans le cadre des phrases où elles apparaissent » [Le Pesant D. \& Mathieu-Colas M., $1998: 6]$.

Dans une approche de ce type, qui s'inscrit contre la partition lexique / grammaire, l'unité prise en considération ne l'est pas en tant qu'élément constitutif d'un système lexical mais en tant que constituant d'un syntagme, ce qui revient à dire que le lexème $e^{6}$, notion opératoire pour le lexicologue, n'est pas au centre de ces travaux. En effet, ces derniers ont pour horizon la description des actualisations discursives diverses, 
contrainte elle-même par les exigences du traitement automatique qui suppose l'établissement de $"$ tables $»^{7}$ ou de "classes d'objets ${ }^{8}$, pour rendre compte de manifestations sémantiques et/ou morphologiques complexes - considérées par les lexicologues comme inhérentes au fonctionnement du lexique - induites, notamment, par un phénomène «typique du langage naturel qu'est la polysémie » [Martin R., 1972:125] et par « une propriété des langues naturelles dont l'importance a été méconnue pendant très longtemps » [Gross G., 1996: 5], le figement. Aussi l'objectif poursuivi ne semble pas correspondre à la mise au jour du système lexical dans son entier, par exemple à l'établissement, de manière inductive - à partir des relations qui structurent les microsystèmes - des grandes lois qui régiraient la structuration de ce système. On a, dans la ligne de Harris, une description minutieuse qui ne se présente pas comme la vérification d'une hypothèse théorique généralisante sur le fonctionnement global du lexique. Si le lexicologue à l'instar de Benveniste considère également que :

« le 'sens' d'une forme linguistique se définit par la totalité de ses emplois, par leur

distribution et par les types de liaisons qui en résultent » [Benveniste E., 1966:290],

et donc étudie aussi les micro-systèmes reflétant l'ensemble des emplois définis en termes distributionnels et en termes syntaxiques, il cherchera, en outre, en s'appuyant éventuellement sur les propriétés morphologiques, à mettre au jour la relation qui pourrait être établie entre les diverses acceptions grâce, par exemple, à l'établissement d'un signifié de puissance (comme le propose J. Picoche dans une perspective guillaumienne), ou en faisant le départ entre propriétés intrinsèques et propriétés extrinsèques (comme le suggèrent $\mathrm{P}$. Cadiot \& $\mathrm{F}$. Nemo [1997: 24-34]) et à établir des corrélations avec les autres unités lexicales afin de contribuer à l'élaboration d'une explicitation systématique des phénomènes lexicaux.

9 Hormis cette perspective dictionnairique apparait, dans les explorations actuelles, une autre orientation dominante qui est susceptible d'être liée à la première : les recherches terminologiques, souvent associées à la néologie ${ }^{9}$. Si pour certains spécialistes la terminologie est étroitement liée à la lexicologie, pour d'autres il s'agit de disciplines différentes opérant sur des unités de base distinctes :

«La lexicologie s'occupe de l'étude des mots, la terminologie de l'étude des termes »[Cabré M.-T., 1998: 75].

La distinction établie fréquemment entre mot et terme ${ }^{10}$ témoigne d'approches différentielles - sémasiologique ou onomasiologique - du même objet puisque le terme est nécessairement un mot, une unité lexicale, et tout mot est susceptible d'accéder au statut de terme à l'intérieur d'un univers référentiel étroitement délimité en fonction de critères extra-linguistiques d'ordre notionnel. Dans cette perspective, l'objectif ${ }^{11}$ est de rendre compte d'une organisation lexico-dénominative au sein d'un domaine et non de décrire linguistiquement l'ensemble du système. Le terminologue est amené à définir le contenu du terme par une procédure référentielle ou dénotative, alors que pour le lexicologue ce terme, appréhendé en tant qu'unité lexicale, recevra son identité de sa place dans le système, i.e. de ses interrelations avec les autres unités sur les plans sémantique aussi bien que formel (morphologique ou syntaxique).

11 Les deux champs à visée pragmatique que nous venons d'évoquer ont donc pour matière première l'unité lexicale qui est susceptible d'être désignée par les termes mot, donnée lexicale ou, dans le cadre des vocabulaires de spécialité, unité terminologique, terme. Même si le syntagme nominal unité lexicale est en usage dans ces discours, la notion qui s'y rapporte n'est pas considérée comme opératoire ${ }^{12}$ en tant qu'unité du lexique. Il s'agit 
plutôt d'une étiquette qui se prête à la désignation d'une unité graphique (suite de lettres précédée et suivie d'un blanc) ou plus généralement d'un groupe d'unités graphiques (de mots graphiques) correspondant à une unité sémantique (une unité signifiante) dont l'identité est à construire dans la dépendance d'une unité plus vaste : la phrase pour les uns, la nomenclature pour les autres. On pourrait émettre l'hypothèse que les lexicologues se démarquent des "néo-lexicographes", i.e. les tenants du lexiquegrammaire, qui ont une approche essentiellement syntagmatique, et des terminologues, qui privilégient la dimension paradigmatique, par une interprétation différente du syntagme unité lexicale qui pour les premiers est classifiant (s'oppose à unité grammaticale) et pour les autres qualifiant (élément de l'unité phrastique, ou d'un répertoire terminologique). Ce qui permet d'envisager que le syntagme unité lexicale serait susceptible d'acquérir une valeur dénominative en lexicologie (formerait une lexie complexe $^{13}$ ) et conserverait une valeur désignative (relèverait de la collocation ${ }^{14}$ ) dans les deux autres approches.

En définitive, l'unité lexicale évoquée par les tenants du lexique-grammaire correspond au mot et sert de point de départ à l'observation de l'emploi (voire des emplois), elle n'a donc qu'un statut instrumental : on étudie, par exemple, les constructions et distributions de prendre ou de avoir beau. Conçue isolément elle n'est pas pertinente : elle ne le devient que dans la phrase qui en fait surgir l'identité. Pour le terminologue, l'unité lexicale n'est digne d'intérêt qu'en fonction de sa potentialité terminologique, sa capacité à devenir un terme. Il s'agit essentiellement de noms susceptibles d'acquérir institutionnellement ${ }^{15} \mathrm{le}$ statut de dénomination. Le lexème, qui est pour le lexicologue l'unité lexicale par excellence, est pertinent et opératoire, il relève du système de signes que constitue la langue, c'est-à-dire que son signifié est une valeur qui ne se confond ni avec le référent ni avec l'idée qu'on en a intuitivement.

\section{De l'utilité du syntagme unité lexicale}

13 Malgré une appréhension différente de la notion d'unité lexicale et donc de son utilisation, tous ces travaux se heurtent, à partir d'un point de vue théorique et/ou pratique, à la même nécessité de circonscrire une unité qui soit à la fois formellement et sémantiquement autonome (par opposition au morphème lié) - ne serait-ce que pour en observer ses emplois. La distinction unité graphique / unité linguistique, évidente sur le plan théorique pour tout linguiste, n'est pas d'une mise en œuvre aisée, notamment parce qu'elle implique une délimitation qui peut varier en fonction des perspectives de recherche, du contexte et de la période prise en compte. Les lexicographes traditionnels privilégient dans leur nomenclature l'unité graphique aux dépens de l'unité linguistique lorsque des formes composées apparaissent - hôtel de ville, par exemple, ne fait pas l'objet d'une entrée spécifique dans le Nouveau Petit Robert, il est défini à l'occasion de l'article « Hôtel ».

14 La mise au jour de la plus petite unité pourvue d'un sens, le morphème, ne résout pas totalement le problème dans la mesure où elle ne satisfait pas systématiquement les critères d'autonomie évoqués précédemment. Le morphème, lorsqu'il n'est qu'une composante d'un lexème, n'est pas toujours "présentable et manipulable" isolément, en particulier quand on a affaire à des mots complexes non construits ${ }^{16}$. Si l'on reprend l'un des exemples traités par D. Corbin, royaume, il parait en effet peu économique pour le locuteur qui consulte un dictionnaire de disposer d'une entrée -aume et peu pertinent 
pour le lexicologue de considérer "aume» comme une unité du lexique puisque «le segment -aume ne se retrouve nulle part ailleurs avec les mêmes propriétés » [Corbin D., $1987: 12]$.

15 Aussi, dans une perpective lexicale, l'usage du terme lexème permet d'éviter une fragmentation improductive ; cependant, défini comme « le mot ou ce qu'il en reste, une fois dépouillé de ses affixes »[Picoche J., 1992: 18]17 ou de manière contrastive par rapport à vocable qui serait le «lexème actualisé dans un discours» [Mortureux M.-F., 1997 : 191], il est d'usage délicat. Dans la première configuration, on est confronté à la mise au jour d'unités difficilement exploitables - comme le démontre J. Picoche - qui correspondent à ce qu'on appelle des racines ou des radicaux ou encore des bases. C'est-àdire à des unités qui dans bien des cas ne sont pas autonomes formellement et prennent un sens différent lorsqu'elles sont associées à tel ou tel affixe : par exemple le radical mass - produit massivement, massif, mais également masselotte, massette (instruments) ou encore massique, massé,sans que l'on puisse en synchronie considérer que le sens du mot soit perçu systématiquement comme compositionnel, réductible à l'addition du sens du lexème avec le sens des suffixes. Lorsqu'on présente le lexème dans son rapport au vocable, comme nous y convie M.-F. Mortureux, on lui donne le statut d'unité dénominative, ce qui permet de le distinguer du grammème (de l'unité grammaticale) en vertu de sa potentialité à référer à un élément de l'univers. L'unité de signification, ainsi délimitée, serait donc d'un niveau supérieur à celle envisagée précédemment (par A. Martinet et J. Picoche) dans la mesure où le morphème lexical, comme le mot, sont susceptibles de n'en représenter qu'une composante. Le lexème, défini par sa relation privilégiée à une réalité tangible ou intangible, devient l'objet constitutif de la spécificité de la démarche lexicologique par rapport aux approches morphologique et/ou syntaxique. Toutefois, cette dénomination est restrictive puisqu'elle est marquée par le trait virtuel qui l'oppose au vocable, ce qui lui donne un caractère abstrait, donc un statut trop particularisant pour désigner n'importe quelle unité lexicale dans n'importe quel contexte.

16 Ces diverses spécifications expliquent le recours fréquent, y compris par les lexicologues, au terme mot parfois associé à un adjectif tel que graphique, comme le propose par $\mathrm{J}$. Picoche [1992 : 23] laquelle réserve l'emploi absolu (mot), à «l'unité de fonctionnement » subsumant ainsi «les unités graphiquement simples mais morphologiquement complexes » et « les unités lexicales graphiquement complexes ». De manière identique, mais en se plaçant d'un autre point de vue, M.-F. Mortureux [1997 : 13] l'utilise « pour désigner l'unité lexicale à valeur dénominative ", et le présente comme un générique permettant de neutraliser la dichotomie lexème / vocable quand celle-ci s'avère non pertinente. Quant à J. Rey-Debove, qui retient le terme morphème pour le «morphème lexical lié », elle réserve mot pour la désignation du «morphème lexical libre » [1998: 227].

Cependant le polysème mot occulte la différenciation particulièrement opératoire entre mot graphique - voire mot phonique, lequel pose le problème de manière encore plus aiguë - et unité significative dans le cas, notamment, de composés syntagmatiques du type hôtel de ville - à propos duquel on peut dire qu'il est composé de trois mots $(N+$ prép. $+N)$ et simultanément qu'il correspond à un mot (mairie). On ne peut en effet exclure du système lexical, soustraire de l'inventaire du stock lexical d'une langue, des unités formellement complexes sous prétexte qu'étymologiquement elles ont pour composantes des unités simples avec lesquelles elles entretiennent des rapports plus ou moins lâches - l'étranger qui s'adresserait à l'hôtel de ville dans l'espoir d'y passer quelques nuitées serait sans 
doute surpris par l'accueil qui lui serait réservé. Néanmoins, l'établissement des critères de délimitation pour les unités supérieures au mot demeure problématique d'autant qu'elle est contrainte par des variations historiques - le figement est une opération qui s'inscrit dans la durée - et contextuelles. Par exemple bras droit correspond soit à deux unités lexicales (Son bras droit est engourdi), soit n'en forme qu'une (Son bras droit est dégourdi). En adoptant une perspective diachronique, on constate, à propos du second emploi de bras droit, qu'il est difficile de parler d'un mot formellement nouveau puisqueles composants de base existaient antérieurement de manière autonome (autonomie fonctionnelle) donc la nouveauté ne réside que dans leur association « mentale » - en l'absence d'indice syntaxique et/ou graphique conviant à l'amalgame -, dans la décision, guidée par l'inscription en contexte, de considérer qu'il s'agit, conjoncturellement, d'une unité lexicale en vertu de l'unicité sémantique dénotative vérifiable par le test de commutation (Son adjoint est dégourdi).

Ce cas illustre le fait que l'unité lexicale est une construction a posteriori - et non une donnée concrète, immédiate - indispensable néanmoins pour aboutir à une description cohérente du système lexical qui doit rendre compte, en l'occurrence, de l'existence de trois unités : bras, droit, bras droit et des relations sémantiques qu'elles sont susceptibles d'entretenir entre elles et avec les autres lexèmes. En outre, on ne peut déduire mécaniquement de cet exemple que le nombre d'unités lexicales est plus important que le nombre de mots, puisque la notion d'« unité » qui présuppose un lien entre forme et sens devrait se manifester par une neutralisation des variantes flexionnelles, ce qui revient à considérer cheval et chevaux comme une seule unité. Si la majorité des unités lexicales correspondent à des mots, on sait que tout mot ne constitue pas inéluctablement une unité lexicale (comme l'atteste l'exemple, désormais classique, de fur dans au fur et à mesure).

Après avoir rendu compte sommairement des rôles dévolus à la notion d'unité lexicale au sein de démarches dont les visées sont dissemblables, et avoir tenté de la définir comparativement à lexème et mot, on s'aperçoit que le recours à cette dénomination permet d'éviter mot qui est trop imprécis et lexème qui est trop restrictif, dans la mesure où on ne peut l'utiliser pour désigner un vocable, une unité lexicale en discours. Il n'en demeure pas moins que toute création lexicale ne devient pas nécessairement une unité lexicale, c'est-à-dire une composante du lexique, comme le souligne L. Guilbert toutes les «formations ne pénètrent pas dans le lexique de la langue, mais elles exercent sur lui une pression constante » [1975: 52]. Ainsi en est-il, notamment, des mots possibles proposés par D. Corbin, de certains mots d'auteur, de certaines propositions émanant des journalistes telles que "Euroland(e)», qui fit l'objet, récemment, d'une controverse. Il semble que, pour comprendre le fonctionnement du lexique, il soit nécessaire de s'interroger sur l'intégration et les exclusions de certaines créations ou productions de type lexical.

\section{Manifestations et facteurs de lexicalisation}

Pour explorer le processus de formation de l'unité lexicale, i.e. son inscription dans le lexique, on examine certaines réalisations discursives assimilables lors de leur émergence à un néologisme :

«Un néologisme est un mot reconnu à la fois comme nouveau et susceptible de se lexicaliser. Car on l'applique essentiellement à des mots en cours de diffusion, avant 
que leur diffusion n'ait abouti à les faire enregistrer dans les dictionnaires généraux. Quant aux créations lexicales littéraires, qui n'ont pas, sauf exception, vocation à se lexicaliser, on les appelle plutôt des hapax (emploi unique), pour signaler leur appartenance exclusive au vocabulaire, voire au style d'une œuvre ou d'un auteur » [Mortureux M.-F., 1997 : 105]

21 Cette définition met en exergue l'instabilité du mot nouveau qui peut, bien qu'il fût ou soit encore en usage, ne pas s'intégrer au lexique (juppette ${ }^{18}$ ou balladurette $e^{19}$ ), ou au contraire devenir une unité lexicale (eurogrève), et présente l'insertion dans les dictionnaires comme indice de lexicalisation. La non-intégration dans la nomenclature d'ouvrages lexicographiques est utilisée par un grand nombre d'auteurs - M -T. Cabré (1998), L. Guilbert (1975), J. Rey-Debove (1971), etc. - comme critère néologique, indice de pré-lexicalisation; cependant si l'on considère que les dictionnaires reflètent un état de la langue à un moment donné et que par conséquent ils sont de précieux témoins ${ }^{20}$, on ne peut simultanément les pourvoir d'un rôle normatif et prescriptif. Ce qui revient à dire qu'ils sont des médiateurs avisés mais qu'ils n'interviennent pas explicitement dans le processus. Rechercher les fondements de la lexicalisation, les facteurs qui contribuent à l'accession à ce statut ne relève pas de leur mission.

\section{III.1. Les marqueurs en question}

Les manifestations de la lexicalisation sont donc repérables grâce à un usage récurrent, à une observation minutieuse des fréquences d'emploi, qui motivera l'insertion dans les dictionnaires. Il n'en demeure pas moins que ce mode de repérage est aléatoire puisqu'aucun ouvrage lexicographique n'est exhaustif, que le nombre d'entrées ne constitue pas une garantie absolue, car un item tel que beur, beurette est absent du Trésor de la Langue Française (désormais TLF) mais se trouve dans le Nouveau Petit Robert (NPR) et le Petit Larousse Illustré (PLI). Certes l'absence, pour une unité lexicale récente, est susceptible d'être justifiée par la périodicité des remises à jour des dictionnaires usuels la date d'attestation proposée par le NPR à propos de beur est 1980 alors que le volume IV du TLF, concerné par cette éventuelle insertion, date de 1975. Cependant l'intégration d'un item dans ces ouvrages est également liée à l'intuition du lexicographe qui se doit de spéculer sur la pérennité d'une nouvelle réalisation lexicale. Témoigne de ces variations subjectives la non-identité des nomenclatures du NPR et du PLI; par exemple, feuj est mentionné par le NPR mais écarté par le PLI, alors que ce dernier consacre un article à keum qui ne figure pas dans le NPR. En outre, interviennent dans les décisions de ce type des considérations idéologiques et commerciales. Par exemple, aucun de ces dictionnaires ne peut faire l'impasse des recommandations officielles parues au Journal officiel, pourtant des propositions de nouvelles dénominations comme listage pour listing ou marchandisage n'ont guère rencontré fortune.

Se fonder sur l'usage pour décider de la lexicalisation d'une unité suppose que le recueil de données se fasse sur une période relativement longue pour être fiable et présuppose également que soit exclue du lexique, que perde le statut d'unité lexicale, toute unité n'étant plus en usage. On peut prendre pour exemple des emprunts tels que pérestroika, ou dazibao - présents dans le $N P R^{21}$, dans le $P L I$, mais absents du $T L F^{22}$ - dont la fréquence d'emploi à une période donnée impliquait leur francisation graphique, favorisait l'attribution des marques morpho-syntaxiques propres à notre système (des dazibaos) et permettait d'envisager leur intégration dans notre stock lexical au même titre que mazout et zen, dont les origines sont identiques. Cependant ils sont sortis de l'usage 
contemporain, de même que juppette et balladurette (absents des trois dictionnaires pris pour référence), ils ont acquis une dimension archaïque, comme en témoignent les guillemets qui les accompagnent lorsqu'ils resurgissent dans un texte contemporain. Ainsi, peut-on considérer que ce signe typographique à travers sa fonction autonymique est susceptible de constituer un indice de non-lexicalisation (néologisme) ou de "délexicalisation » (archaïsme). Toutefois, la corrélation opérée de manière systématique entre l'absence de guillemets et la lexicalisation est contestable, puisque l'on sait qu'un locuteur peut forger un néologisme à son insu en mobilisant (ou non) les ressources du système sans utiliser, de marques spécifiques et qu'inversement une unité lexicalisée qui serait inconnue d'un interlocuteur sera appréhendée par celui-là comme une unité étrangère à sa compétence lexicale et de ce fait reprise éventuellement entre guillemets. Nous ne disposons donc que d'indices (insertion dans des dictionnaires généraux, fréquence d'emploi, absence de guillemets, compétence linguistique) et non de marqueurs absolus d'intégration dans le système lexical. Apprécier le degré de lexicalisation d'une unité pose un problème corollaire au repérage d'un néologisme qui " dépend d'un jugement relatif et même subjectif, lié à sa définition même, qui repose non pas sur la nouveauté objective, mais sur un sentiment de nouveauté » [Rey A., 1988 : 282]. Cependant, malgré la difficulté à porter un jugement définitif sur la lexicalisation de certaines formations lexicales, notre expérience de locuteur francophone nous permet, par exemple, de savoir que chauffe-savates, canon à patates, éventre-tomates et écorche-poulet forgés par B. Vian [Complainte du progrès] ne sont pas entrés dans l'usage, alors que surréaliste attribué à $\mathrm{G}$. Apollinaire a été adopté. Ces exemples tendent à prouver également que la source (susceptible d'être littéraire ou non, voire législative ou non) n'intervient pas de manière significative dans le processus d'intégration.

\section{III.2. En quête des facteurs de lexicalisation : vérification de quelques hypothèses}

L'évocation d'inclusion ou d'exclusion de certaines formations lexicales incite à s'interroger sur les facteurs qui contribuent à cette sélection «naturelle ", de manière à mieux cerner le fonctionnement du système.

Le départ entre l'influence des facteurs externes et des facteurs internes à la langue est sans doute une des premières investigations à mener dans la mesure où a priori toute dénomination implique un rapport privilégié entre une unité lexicale et ce qu'elle est susceptible de désigner puisque «si l'on accepte que parler, c'est dire quelque chose, le réel est alors partie prenante dans le commerce linguistique » [Kleiber G. 1997 : 9]. Ainsi, pourrait-on considérer que les hapax de B. Vian, cités ci-dessus, ne se sont pas implantés parce qu'ils étaient dépourvus de référents tangibles. Toutefois, cette analyse n'est pas généralisable et repose sur une confusion, fréquente, entre existence et référence, à laquelle s'opposent les dénominations d'objets imaginaires qui ont été intégrées : le père Noël, la licorne, le dahu, les soucoupes volantes, etc. Ces exemples tendent à prouver que le référent peut être le résultat d'une représentation construite par un discours et que par conséquent l'existence tangible n'est pas prédominante. En outre, milite en faveur de l'écartement du critère référentiel les attestations synonymiques qui par définition sont co-référentielles. Si l'on choisit pour illustrer cela le paradigme inatteignable, inaccessible et inattingible, on s'aperçoit que ces unités ne sont pas toutes lexicalisées, en effet la troisième est hors d'usage, extérieure à la compétence synchronique des locuteurs alors 
qu'il s'agit de la seule forme reconnue par les puristes qui, malgré l'absence de critères morphologiques ${ }^{23}$, n'admettent pas inatteignable sous le prétexte qu'il correspond à un doublon impropre, ce qui revient à dire qu'il doit être exclu du lexique.

On ne peut donc relier de manière systématique la question d'intégration à l'existence de ce que l'on veut désigner, car nommer c'est faire exister, et accepter qu'il y ait préexistence de la chose pour valider la dénomination supposerait qu'il y ait prééminence de la réalité matérielle sur la langue, ce qui reste à démontrer. Il est, en revanche, probable que c'est en fonction d'un besoin nouveau, de la nécessité de désigner un nouvel élément, ou de désigner autrement un élément existant, qu'une unité lexicale jusqu'alors inconnue surgit. L'innovation sur le plan de la langue se manifeste alors soit par la création d'un nouveau signe, soit par modification d'un signe existant, i.e. par un réaménagement des associations signifiant/signifié - signifiant en usage auparavant, mais associé à un autre signifié (néologie sémantique: conséquent correspond, aujourd'hui, à considérable), ou nouveau signifiant relié à un signifié ancien (néologie formelle: SDF pour sans-abri). On formule, donc, l'hypothèse que si l'émergence d'une unité est partiellement ${ }^{24}$ liée à des facteurs extra-linguistiques, sa lexicalisation, son insertion dans le lexique serait conditionnée par le système lexical en vigueur.

Cette approche présuppose qu'un terme soit forgé conformément aux règles en usage pour être rapidement lexicalisé et que seule cette conformité lui assure son insertion dans le lexique. Cependant, les néologismes d'auteur comme les mots possibles envisagés par D. Corbin sont bien formés mais non lexicalisés, donc ce critère s'avère insuffisant. De plus, certaines créations qui ont été jugées non conformes aux règles de constructions classiques (dérivation et composition) sont intégrés. Ainsi le $N P R^{25}$ insère dans sa nomenclature des verbes, tels que briefer, positiver, positionner, des adjectifs comme basique , digeste, et des noms club-house, en les faisant suivre de la mention "critiqué », sans indiquer les auteurs ni les motifs de la réserve. Il s'agit pour la plupart de mots empruntés à l'anglais ${ }^{26}$ auxquels on reproche habituellement de ne pas respecter le système phonographique du français et de créer ainsi «des difficultés » supplémentaires à «l'usager moyen " [Goosse A., 1993 : 193]. Ces considérations, dans le cas présent, ne concernent que briefer qui nécessite que l'on considère le -e- précédant le -f-comme un « e muet " (situation analogue à celle rencontrée dans des occurrences françaises, telles que remerciement) et club-house qui conserve en effet des traces de son étrangeté, mais dont les équivalents recommandés officiellement ${ }^{27}$ : pavillon, maison de club n'ont eu aucun succès. Ainsi l'usager a plébiscité des anglicismes aux dépens d'unités lexicales plus conformes aux règles morphologiques française, plus motivées, mais qui ont cependant l'inconvénient pour le premier d'être trop générique, ressenti comme un hyperonyme effaçant la spécificité, et pour le second d'être long et peu évocateur dans la mesure où il s'inscrit dans un paradigme très fourni au sein duquel le syntagme prépositionnel spécifie : une localisation géographique (maison de campagne), un volume par le biais d'une catégorisation prototypique des habitants (maison de maitre vs maison de poupée) ou encore les matériaux de construction (maison de verre) ou enfin la destination (maison de retraite, maison de repos, maison d'arrêt).

Norme et lexicalisation ne vont donc pas de pair, sauf à prendre norme dans le sens promu par J. Rey-Debove :

«Le lexique comprend des unités d'importance inégale à cause de l'emploi qu'en font les locuteurs. Cette somme des emplois particuliers s'appelle la «norme » [ReyDebove J., 1998 : 214]. 
Cette «norme » définie comme générale et concrète se trouve inséree dans une structure ternaire et se distingue du «système » qui est général et abstrait, et de la " parole» qualifiée de singulière et concrète. La tripartition proposée, qui a pour vocation de se substituer à la dichotomie saussurienne langue/parole, permet de situer l'unité lexicale au niveau de la norme, c'est-à-dire d'établir une distinction entre n'importe quel fait de parole et la langue. Ainsi pourrait-on proposer en extension de cette nouvelle répartition trois types de réalisations lexicales: le néologisme (unité non lexicalisée) en tant que réalisation concrète singulière, l'unité lexicale en tant que réalisation concrète et d'usage général, et le lexème en tant qu'unité abstraite ; étant entendu que le néologisme - que l'on pourrait appeler xénisme ou pérégrinisme, si ces termes n'étaient pas dévolus à la désignation d'emprunts conjoncturels à une langue étrangère - est susceptible de changer de statut, de venir enrichir le stock lexical de la langue, et que l'unité lexicale renvoie inévitablement à un lexème. Cela permettrait de différencier les unités qui ont été intégrées à l'usage, et corollairement à la langue, de celles qui demeurent dans «l'antichambre» indépendamment de leurs modes de constitution, qu'elles soient formées ou non suivant les règles en vigueur. La discrimination, instaurée ainsi, ne repose pas sur un jugement esthétique, qui pourrait être celui émis par des puristes ${ }^{28}$, mais préserve la possibilité d'exclure un assemblage de graphèmes ou de phonèmes - par exemple : *infractus pour infarctus ou *aréoport pour aéroport - qui serait « contraires aux structures de la langue » [Goosse A., in Leeman D., 1994 : 11], donc ne pouvant accéder au statut d'unité lexicale.

31 Toutefois, il reste à définir de manière rigoureuse les structures lexicales du français. Partant du constat de J. Rey-Debove :

«Malheureusement, la norme entérine quantité de mots impossibles selon le système qui ne sont pas les moins viables » [1998:217],

on peut envisager que le problème se situe au niveau de notre conception du système et non au niveau du système. On a reproché aux grammairiens traditionnels la faible productivité des règles édictées puisqu'elles nécessitaient l'établissement d'une liste d'exceptions, il serait regrettable que nous tombions dans le même travers, alors que nous disposons d'une théorie plus rigoureuse, d'un plus grand nombre de moyens techniques, et de données informatives plus importantes.

Ainsi, le «système » de référence semble ne prendre en compte que les affixations et les compositions, notamment savantes, et exclure les " unités marginales » [Rey-Debove J., 1998 : 213] parmi lesquelles on trouve les emprunts, les sigles, les troncations et les motsvalises. Si l'on adopte un point de vue descriptif et non prescriptif, on constate que ces dernier procédés ont pour inconvénients de déroger au principe de la motivation et que, de ce fait, ils accroissent le nombre d'unités de base. Toutefois, l'arbitrarisation du signe ne peut constituer une anomalie par rapport au système linguistique. Les travaux en morphologie mettent en évidence, à travers les notions de mot complexe non construit et de mot simple, le fait que la motivation n'est pas généralisable, et que les mots construits acquièrent souvent un sens conventionnel non réductible au sens prédictible. On ne reviendra pas ici ${ }^{29}$ sur le détail de ces procédures - sur le fait qu'il n'existe pas davantage de langue pure que de race pure, que les sigles, comme certaines troncations relevant de l'abréviation, ont une vertu économique ${ }^{30}$. Même si le caractère hybride de certaines formations (telles que les sigles et les mots-valises ${ }^{31}$ qui conjuguent extension par composition et réduction des composants) ne satisfait pas notre désir de rationalité, elles 
sont extrêmement productives et à ce titre ne peuvent être mises raisonnablement en marge du système lexical.

En revanche, est plus controversée l'intégration de certaines unités forgées à partir du verlan $^{32}$, car il ne s'agit pas d'adopter le principe de ce codage comme une procédure généralisable constitutive de notre système lexical ${ }^{33}$. Pourtant, parmi les termes récemment insérés dans les dictionnaires, on trouve par exemple : beur, beurette qui figurent dans le PLI, NPR (1994), comme dans Le Robert électronique (1996), munis d'une date d'attestation (1980) et de la mention «FAM». On remarque que cette nouvelle unité lexicale qui relevait d'un registre dit «familier» a changé de statut, comme pourrait en témoigner son usage régulier dans des échanges ou des écrits soutenus, par exemple dans un mensuel, tel que Le Monde diplomatique ${ }^{34}$. L'intégration de plain-pied dans le système lexical peut se justifier par le fait que cette dénomination vient « combler une absence, suturer un vide» pour paraphraser G. Petit [1998: 29], car l'équivalent en registre standard nécessite le recours à une périphrase : Maghrébin né en France de parents immigrés. Il n'en demeure pas moins que parmi les produits du verlan mentionnés par les dictionnaires précités, on constate une diversité du degré de lexicalisation dans la mesure où pèse sur l'emploi de certains une restriction liée à la situation de communication. En effet, il semble actuellement difficile de mettre sur le même plan que beur, ripou, verlan voire barjo(t) et loubard, les réalisations telles que meuf (1981), feuj (1988), keuf (1978) et béton (relié à Laisse béton qui a pour date d'attestation 1970), ces derniers appartenant exclusivement, comme tous les autres mots forgés à partir du verlan, « au registre parlé, familier, jeune, utilisé dans une situation de discours informelle et surtout dans le groupe de pairs » [Méla V., 1997 : 29]. La banalisation des cinq premiers termes, par opposition aux quatre derniers, permet d'émettre des hypothèses à propos des processus de lexicalisation. Si verlan se trouve dans une situation analogue à celle de beur, i.e. vient combler un vide, ce n'est pas tout à fait le cas des autres termes qui sont présentés comme entretenant des relations synonymiques avec des unités lexicales standards qui leur servent d'étymon : jobard, balourd, pourri. Cependant, on peut considérer que ripou a subi une restriction de sens liée à une affectation référentielle spécifique puisqu'il désigne un policier malhonnête, et envisager qu'il a acquis ses lettres de noblesse ${ }^{35}$ grâce à son emploi cinématographique, de même loubard a une fonction dénominative ${ }^{36}$ alors que son "étymon» balourd est qualifiant. Ils ne sont donc pas sémantiquement identiques, mais la version «étymologique » est susceptible de motiver la dénomination. Quant à barjo qui, d'après V. Méla, «n'est plus reconnu comme un mot de verlan », mais dont le caractère familier perdure, il sert d'illustration à l'auteur pour avancer la conjecture suivante :

"On ne peut pas vraiment affirmer qu'un mot de verlan s'est lexicalisé tant qu'il n'y

a pas eu un oubli collectif de son origine " [1997:29],

Cette supputation est invalidée, en l'occurrence, si l'on conserve aux dictionnaires leur rôle d' " attesteur ", mais il semble que la lexicalisation atteigne plus facilement un terme forgé en verlan lorsque le produit final relève d'une procédure plus complexe que la simple inversion de syllabes, et qu'ainsi l'étymon est occulté. Verlan ou beur, par modifications orthographiques, voire par troncation syllabique dans le second, ne laissent pas aux locuteurs novices en la matière la possibilité de reconstituer l'unité initiale : * lanver et * reub ne sont pas facilement associables à envers et arabe (qui existe aussi sous la forme rebeu), avec lesquels d'ailleurs ils n'entretiennent pas de relations synonymiques étroites. Ces exemples tendraient à prouver que la conjonction des écarts formels et 
sémantiques par rapport à "l'existant " serait un facteurs favorisant la lexicalisation. Confirmerait cette hypothèse le fait que des réalisations actualisées fréquemment - telles que tromé qui est synonyme de son étymon métro - ne sont pas intégrées dans les nomenclatures des dictionnaires, donc ne sont pas considérées par les lexicographes comme lexicalisées.

En revanche meuf, feuj, keuf figurant dans le NPR, ou keum introduit comme item dans le PLI, sont non seulement co-référentiels à leurs équivalents non verlanisés : femme, juif, flic , mec, mais encore se présentent comme des synonymes dont l'usage est normé, restreint, connotatif. Leur présence dans des dictionnaires usuels dits généraux, alors qu'il s'agit d'usages particuliers, témoigne du fait que cette parlure « accède incontestablement, par la grâce d'une médiatisation régulière, à une certaine respectabilité » [Boyer H., 1997 : 13] et semble relever davantage d'une opération commerciale que d'une nécessité linguistique ; sinon, comment justifier l'absence de zarbi (bizarre), reum (mère), etc., que l'on entend fréquemment, y compris, dans le milieu estudiantin, mais que l'on ne pourrait utiliser dans une situation un peu formelle?

Si on ne répertoriera pas l'inversion des syllabes parmi les procédures morphologiques disponibles, on ne pourra l'effacer des gloses étymologiques puisque certaines unités lexicales sont les résultantes de la combinaison de l'inversion suivie d'un réaménagement

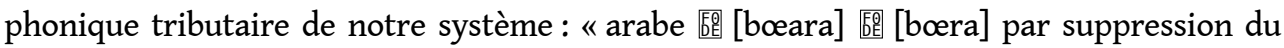
hiatus et finalement 熙 [bœr] par troncation » [Méla V., 1997 : 23].

37 A l'issue de cette première approche s'impose le constat que toute formation de type lexical n'est susceptible de s'intégrer au lexique que si (et seulement si) elle vient combler un vide ressenti comme un manque, à un moment donné, par un grand nombre de locuteurs. La nécessité stimule, donc, création et production lexicale, mais la forme lexicalisée sera conditionnée généralement par la langue, et exceptionnellement par le discours, par les unités lexicales déjà attestées et cela éventuellement aux dépens des règles en vigueur antérieurement à l'apparition d'un néologisme. Ainsi l'usage de sidéen ${ }^{37}$ a été (et s'est) imposé au détriment de sidaïque ${ }^{38}$, qui, contaminé par le contexte d'énonciation, fut perçu comme péjoratif, alors qu'il est plus conforme compositionnellement à ce qu'il dénote.

\section{Conclusion}

Le panorama que nous venons d'esquisser à grands traits voudrait montrer que l'identification et donc la délimitation de l'unité lexicale est incontournable pour toute tentative de description du fonctionnement d'une langue indo-européenne, même si cette unité n'a qu'un rôle instrumental. Ainsi, paraît-il difficile d'exclure a priori de la linguistique les approches lexicologiques. Cependant face à cette masse en constante évolution qu'est le lexique, on est trop souvent amené, de manière inconsciente, à se référer à une représentation figée du système, laquelle se révèle parfois inadéquate puisqu'elle ne permet pas de rendre compte de tous les phénomènes qui se manifestent au niveau de la «norme ». On a vu, par exemple, que l'intégration d'une unité n'est pas constamment dépendante des règles connues. La difficulté de maîtrise du système est liée à la pluralité des facteurs constitutifs, à la diversité des usages, à la nécessité d'établir des coefficients de pondération, mais simultanément elle convie à affiner les outils et les 
méthodes permettant de saisir le fonctionnement de la «norme » afin de comprendre le système dans son entier.

\section{BIBLIOGRAPHIE}

ANSCOMBRE J.-C. \& Leeman D. (1994) « La dérivation des adjectifs en -ble : morphologie ou sémantique? ", Langue française $\mathrm{n}^{\circ} 103$, Larousse, Paris.

BENVENISTE E. (1966) Problèmes de linguistique générale, Gallimard.

BOYER H. (1997) « Nouveau français », « Parler jeune » ou « Langue des cités »? Langue française n - 114, Larousse, Paris.

CABRÉ M.-T. (1998) La terminologie. Théorie, méthode et applications, Armand Colin, Ottawa.

CORBIN D. (1987) Morphologie dérivationnelle et structuration du lexique, Presses universitaires de Lille.

COSTE D. (1988) « La situation de la lexicologie dans les rapports entre linguistique et linguistique appliquée », DRLAV n³9, Université Paris III.

CUSIN-BERCHE F. (1998) Le management par les mots. Etude sociolinguistique de la néologie, L'Harmattan, Paris.

DUBOIS J. et alii (1994) Dictionnaire de linguistique et des sciences du langage, Larousse, Paris.

FRADIN B. (1996) «L'identification des unités lexicales », Sémiotiques n 11, Didier-érudition, Paris.

GROSS G. (1986) « Les constructions nominales et l'élaboration d'un lexique-grammaire » Langue française $n^{\circ} 69$, Larousse, Paris.

GROSS G. (1996) Les expressions figées en français, Orphys, Paris.

GUILBERT L. (1975) La créativité lexicale, Larousse, Paris.

HUMBLEY J. (1996) « La légitimation en terminologie », Sémiotiques n 11, Didier-érudition, Paris.

KLEIBER G. (1997) « Sens, référence : que faire de l'extra-linguistique ? », Langages nº 127, Larousse, Paris.

LEEMAN-BOUIX D. (1994) Les fautes de français existent-elles?, Seuil, Paris.

LE PESANT D. \& MATHIEU-COLAS M. (1998) « Introduction aux classes d'objets » Langages n 131, Larousse, Paris.

LERAT P. (1990) «L'analyse morphologique des termes nouveaux » La banque des mots nº spécial, Conseil international de la langue française.

MARTIN R. (1972) «Esquisse d'une analyse formelle de la polysémie » Travaux de linguistique et de littérature $\mathrm{X}$.

MÉLA V. (1997) «Verlan 2000 », Langue française n 114, Larousse, Paris.

MOESCHLER J. \& Auchlin A. (1997) Introduction à la linguistique contemporaine, Colin, Paris. 
MORTUREUX M-F. (1997) La lexicologie entre langue et discours, SEDES, Paris.

PETIT G. (1998) « Approche lexicale et sémantique du vocabulaire familier » Cahiers de lexicologie $\mathrm{n}$

- 72, Didier-érudition, Paris.

PICOCHE J. (1992) Précis de lexicologie française, Nathan, Paris.

POTTIER B. (1985) Linguistique générale, Klincksieck, Paris.

REBOUL S. (1995) « Le vocabulaire des apprentis : entre mot et terme » LINX nº spécial, Université Paris X-Nanterre

REY A. (1988) « Dictionnaire et néologie », Actes du colloque Terminologie et technologies nouvelles, Québec.

REY-DEBOVE J. (1998) La linguistique du signe, Armand Colin, Paris.

\section{NOTES}

1. «L'intérêt de prendre en considération la morphologie en tant que telle est accru par l'absence d'homologie entre le morphologique, le syntaxique et le sémantique » [Lerat P., 1990 : 30]

2. Picoche J. (1977/1992) Précis de lexicologie française, Nathan Université.

3. Lehmann A. \& Martin-Berthet F., (1998) Introduction à la lexicologie, Dunod; Mortureux M.-F. (1997) La lexicologie entre la langue et le discours, Campus, Cedes Nathan ; Niklas-Salminen A. (1997) La lexicologie, Cursus, Armand Colin.

4. Cf., entre autres, les travaux du LADL (Laboratoire d'Automatique Documentaire et Linguistique) et du LLI (Laboratoire Linguistique Informatique).

5. «La première tâche de la lexicologie est de définir son objet propre; pour cela, elle doit dégager de l'ensemble des mots observables dans les discours les unités qui constituent le matériel lexical de la langue [...] Les dictionnaires de langue offrent une représentation, accessible à tout locuteur, du lexique de sa langue. Ils s'inspirent des recherches lexicologiques, dont ils représentent le résultat et reflètent les difficultés, tout en se conformant à des impératifs pratiques [...] Affranchis des contraintes pratiques qui pèsent sur les lexicographes, les linguistes poursuivent l'élaboration d'une description générale et abstraite des phénomènes lexicaux » [Mortureux M.-F., 1997 : 9]

6. "Le lexème est l'unité de base du lexique, dans une opposition lexique / vocabulaire, où le lexique est mis en rapport avec la langue et le vocabulaire avec la parole » [Dubois J. et alii, 1994 : 275].

7. C'est-à-dire de listes rassemblant tous les sens de tous les mots accompagnés de toutes leurs propriétés syntaxiques.

8. «Il s'agit, pour l'essentiel, de classes sémantiques construites à partir de critères syntaxiques, chaque classe étant définie à partir des prédicats qui sélectionnent de façon appropriée les unités qui la composent [...] le modèle s'avère directement opératoire pour le traitement automatique » [Le Pesant D. \& Mathieu-Colas M., 1998 : 6]

9. Cf., notamment, les travaux du centre de terminologie et de néologie : revues Terminologies nouvelles, Ed. RINT (Réseau International de néologie et de terminologie) et La banque des mots, CTN, INaLF, CNRS.

10. «On parlera de mots dans le vocabulaire courant et de termes dans le vocabulaire spécialisé. Dès qu'un mot reçoit un sens particulier, donné par un spécialiste, il est associé à une notion spécifique d'une profession et devient un terme » [Reboul S., $1995: 177]$.

11. «La terminologie et la lexicologie peuvent se distinguer aussi par les objectifs qu'elles poursuivent. Ainsi, la lexicologie, considérée du point de vue de la linguistique théorique, 
s'occupe de mots dans le but de justifier la compétence lexicale des locuteurs. La terminologie, elle, s'occupe des termes pour bien circonscrire la notion » [Cabré M.-T., 1998 : 77].

12. «Le niveau d'analyse minimum est la phrase et non pas le mot [...] Dans une telle perspective, une linguistique du mot n'a donc guère de justification ni même de signification » [Gross G., $1986: 6]$.

13. «La lexie complexe est une séquence en voie de lexicalisation » [Pottier B., 1985 : 266].

14. "On appelle collocation l'association habituelle d'un morphème lexical avec d'autres au sein de l'énoncé, abstraction faite des relations grammaticales existant entre ces morphèmes » [Dubois J. et alii, $1994: 91]$.

15. «La partie terminologique du vocabulaire est le secteur où le contrôle des utilisateurs, ou du moins de certains d'entre eux, s'exerce de la façon la plus explicite et donc la plus visible. Il s'ensuit que les méthodes de légitimation des termes [...] représentent l'essentiel de son originalité » [Humbley J., $1996: 133$ ].

16. «Un mot construit est un mot dont le sens prédictible est entièrement compositionnel par rapport à la structure interne » [Corbin D., $1987: 6]$.

17. Cette définition rejoint celle qu'avait proposée Martinet : "il vaudra mieux désigner comme des lexèmes ceux des monèmes qui trouvent leur place dans le lexique et non dans la grammaire » [Martinet A., $191974: 16$ ].

18. Qui désignait d'abord la procédure de prime pour l'achat d'une voiture neuve, puis les quelques femmes appartenant au gouvernement Juppé. En ce sens, il figure entre guillemets dans un dossier récent consacré «aux femmes célèbres » par ELLE, les guillemets fonctionnant comme marque de non-intégration.

19. Termes encore présents dans Le Monde diplomatique daté d'avril 1997.

20. Le directeur du département de langue française aux éditions Larousse, cité dans Le Monde du 17-18 janvier 1999, précise le rôle des lexicographes «Nous sommes des observateurs; nos dictionnaires sont des miroirs. Il tiennent compte autant de l'usage que des recommandations de l'Académie française ".

21. Les dates d'attestation étant respectivement 1986 et 1970.

22. Le volume VI où devrait se trouver dazibao date de 1978 et le volume XIII où pourrait être inséré pérestroika date de 1988. On ne peut donc invoquer ici le prétexte historique.

23. Comme J.-C. Anscombre et D. Leeman le démontrent [1994: 32-44].

24. Cette restriction a, notamment, pour finalité de souligner que l'émergence d'une nouvelle unité est également liée à un manque, à une "défaillance » linguistique, à un trou du lexique antérieurement construit.

25. Le PLI n'intègre pas : positiver (promu par les publicitaires des magasins Carrefour) ni clubhouse.

26. Hormis l'adjectif digeste formé par aphérèse à partir d'indigeste et le verbe positiver forgé à partir de positif.

27. Arrêté relatif à la terminologie du sport, datant du 18 février 1988, paru au Journal officiel le 6 mars 1988.

28. Dont le postulat est, comme le rappelle D. Leeman, «que toute modification est mauvaise : elle altère la pureté de la langue et est le fait de personnes incultes, qui abâtardissent le français par ignorance de son histoire ou de son génie » [1994:28].

29. Cf. Cusin-Berche F. (à paraître en 1999) "Exploration des caractéristiques des langues de spécialité ... La quête du Graal", collection Champs linguistiques, édit. Duculot.

30. «Le principe de l'économie linguistique repose sur la synthèse entre des forces contradictoires (besoin de communication et inertie) qui entrent constamment en conflit dans la vie des langues » [Dubois J. et alii, $1994: 163$ ].

31. Nous avons proposé pour des formations, comme progiciel (construit à partir de pro(duit lo)giciel), qui ne possèdent pas de « segment commun aux deux bases » et donc ne correspondent 
pas aux critères constitutifs du mot-valise, de les désigner par le terme compocation (compo(sition tron)cation).

32. « le processus de vernalisation consiste, rappelons-le, à inverser les syllabes d'un mot pour en masquer l'identité et en réserver l'usage aux locuteurs familiers du procédé. Cette inversion peut s'accompagner ou non d'une troncation qui rend encore plus difficile l'identification de l'unité employée » [Sourdot M., 1997 : 77].

33. "Les locuteurs du verlan, et ceux qui ne le parlent pas, se demandent souvent si le verlan est encore du français. Nous répondrons oui tout en soulignant les différences voire les déviances qui font que les mots en verlan ressemblent parfois fort peu à leurs homologues français » [Méla V., $1997: 26]$.

34. Voir les diverses occurrences dans les articles suivants : « Un vote contre l'immigration... et l'injustice » (mars 1998, p. 10), « Marseille ou le mythe vacillant de l'intégration » (juillet 1997, p. 6-7), «Splendeurs et misère du 'fast-foot'" (septembre 1996, p. 27), "Grincements du désespoir» (février 1994, p.13), «Le sourire des gorgones» (février 1992, p. 32), «Le rap, complainte des maudits » (décembre 1990, p ; 4-5), «Le roman beur » (janvier 1989, p. 2), «Les cris et les rêves du roman beur » (octobre 1988, p. 18-19), «L'identité française entre archaïsme et modernité » (août 1988, p.18-19), «Le rock, creuset pour une intégration? (août 1988, p. 18-19), «Ceux de la 'deuxième génération'« (août 1985, p. 4-6).

35. Comme est susceptible d'en témoigner la phrase : «Il est assez étonnant de trouver le plur. en $x$ dans des mots du XX ème s. : un ripou, des ripoux », extraite du célèbre Bon usage [1993 : 793].

36. "Jeune homme vivant dans une banlieue, une zone urbaine, appartenant à une bande et affectant un comportement asocial. 崷 loulou, voyou (cf. Blouson noir, mauvais garçon). ABRÈv. LOUB, Une bande de loubs - On rencontre le fém. LOUBARDE » [Nouveau Petit Robert : 1305].

37. «Sidéen, de par sa construction, semblerait mieux convenir à tout malade atteint d'un syndrome immunodéficitaire, quelle que soit son origine (virale ou non)» [TLF, $1992: 465]$.

38. « malade atteint de syndrome immunodéficitaire acquis d'origine virale » [TLF, $1992: 465]$

\section{RÉSUMÉS}

La notion d'unité lexicale n'est considérée comme opératoire ni par les tenants du lexiquegrammaire qui n'appréhendent le lexique que dans sa dimension syntagmatique ni par les terminologues qui privilégient l'axe paradigmatique, en revanche elle se révèle être au centre des travaux des lexicologues qui cherchent à rendre compte du système lexical dans son entier. Ces derniers sont confrontés de ce fait aux distorsions entre la lexicalisation induite par l'usage et le « morphologiquement correct».

Both the supporters of the lexique-grammaire and the terminologists refuse to consider the notion of «lexical unit» operative, the former because they conceive the lexicon as limited to its syntagmatic aspect, the latter because they focus on the paradigmatic-axis. The notion is nevertheless essential in the research of those lexicologists who aim at describing the lexical system as a whole. As a result, such a description is hindered by the discrepancies between use and wont lexicalization and « morphological correctness » 


\section{AUTEUR}

\section{FABIENNE CUSIN-BERCHE}

Université Paris-Sud, CEDISCOR (UPRES SYLED)

28 rue des Fossés Saint-Bernard

75005 Paris

FCUSIN@PARIS3.SORBONNE.FR 